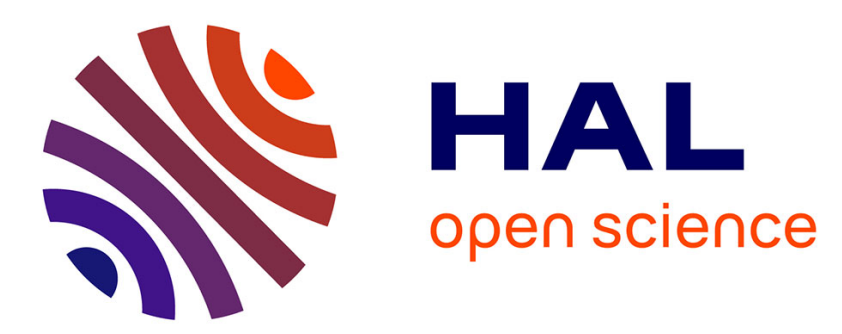

\title{
Polyfonctionnalité et transcategorialité des morphèmes russes vrode et tipa
}

Sergueï Sakhno

\section{To cite this version:}

Sergueï Sakhno. Polyfonctionnalité et transcategorialité des morphèmes russes vrode et tipa. Thierry Ponchon, Hava Bat-Zeev Shyldkrot, Annie Bertin. Mots de liaison et d'intégration: Prépositions, conjonctions et connecteurs, John Benjamins Publishing Company, 2017, 10.1075/lis.34.11sak . halshs01726016

\section{HAL Id: halshs-01726016 \\ https://shs.hal.science/halshs-01726016}

Submitted on 7 Mar 2018

HAL is a multi-disciplinary open access archive for the deposit and dissemination of scientific research documents, whether they are published or not. The documents may come from teaching and research institutions in France or abroad, or from public or private research centers.
L'archive ouverte pluridisciplinaire HAL, est destinée au dépôt et à la diffusion de documents scientifiques de niveau recherche, publiés ou non, émanant des établissements d'enseignement et de recherche français ou étrangers, des laboratoires publics ou privés. 
Serguei SAKHNO, MCF HDR, U. Paris Ouest Nanterre, MoDyCo, ssakhno@u-paris10.fr

\section{POLYFONCTIONNALITÉ ET TRANSCATEGORIALITÉ DES MORPHÈMES RUSSES VRODE, TIPA : FONCTIONNEMENT ET ASPECTS TYPOLOGIQUES}

En russe, les morphèmes vrode, mot à mot «dans le genre de », et tipa, mot à mot « du type de », qui peuvent fonctionner comme prépositions et comme mots discursifs ou connecteurs, présentent des parcours sémantiques proches : on passe de la taxonomie «naturelle » à la « généricité par défaut » ou à la « modélisation ${ }^{1}$. Vrode et tipa apparaissent dans le discours russe comme - marqueurs de comparaison, d'exemplification et d'approximation («hedge » au sens de Lakoff 1973, cf. les marqueurs fr. espèce de, sorte de, genre de, comme, analysés dans Mihatsch 2006, 2007, 2009) ; - marqueurs de modalité épistémique et d'hétérogénéité énonciative (interlocutivité / interdiscursivité).

Une des questions fondamentales que l'on se pose en analysant ces lexèmes polyfonctionnels (voir aussi Bertin, Bat-Zeev Shyldkrot, Soutet 2013): comment rendre compte des nombreuses variantes sémantiques et les divers modes d'utilisation de ces unités dont le sens est éminemment dépendant du contexte?

La transparence de leur forme interne ${ }^{2}$ (vrode $<v+$ rode, cas locatif de $\operatorname{rod}$ 'genre; sorte' ${ }^{3}$; tipa, cas génitif de tip 'type, catégorie', emprunt savant au grec) laisseraient préjuger d'une relative simplicité de leur fonctionnement. Mais cette impression est trompeuse: dans une grande partie de leurs emplois contemporains, vrode n'a pas la valeur 'dans le genre de', et tipa ne signifie pas toujours 'du type de' ${ }^{4}$.

\section{VRODE : DE LA TAXONOMIE «NATURELLE » À LA « GÉNÉRICITÉ PAR DÉFAUT»}

On remarquera d'abord que vrode, contrairement à ce que laisse supposer son étymologie (« dans le genre de ...»), ne peut pas fonctionner comme opérateur de classification, c'est-à-dire qu'il n'a aucun emploi proche de celui de russe rod 'genre' dans par exemple Sofa - rod divana 'Le sofa est un genre / une sorte de divan'. Cf. l'étrangeté de :

(1) Petuški - ??vrode goroda (Gén.), mot à mot : «Petuški est dans le genre de ville ».

Pour un russophone sachant qu'il s'agit d'une ville russe (située à l'est de Moscou), l'énoncé (b) est contraint; en tout cas, il ne peut pas fonctionner avec le sens 'Petuški est une ville / Petuški est incluse dans la classe des villes ${ }^{5}$. En revanche, vrode va apparaître dans deux principaux groupes de contextes qui ne relèvent pas de la classification taxonomique stricto sensu :

(2) Petuški - vrode našego goroda (Gén.), takaja že dyra - 'Petuški, c'est dans le genre de / un peu comme notre ville, un petit trou de province' : ici, vrode a un fonctionnement proche d'une préposition de « comparaison modalisée »;

(3) Petuški - vrode gorod (Nom.), a vid u žitelej sovsem derevenskij - 'Petuški est apparemment / en principe une ville, mais ses habitants ont des allures plutôt campagnardes' : ici, vrode a un fonctionnement

\footnotetext{
${ }^{1}$ Je tiens à remercier Ch. Bonnot (Professeur à l'INALCO) et D. Paillard (Directeur de recherches, U. Paris 7), ainsi que O. Inkova (MCF, U. de Genève) de leurs précieuses remarques et critiques qui ont permis d'améliorer certaines parties de la présente étude et de préciser certaines formulations.

${ }^{2}$ Cette notion est analysée dans certains de nos travaux, voir notamment Sakhno 2001.

${ }^{3}$ Etymologie du substantif russe rod 'genre, espèce, clan, etc.' (< 'naissance, procréation') : racine slave -rod- < i.-eu. *ordh'élevé, grandi, arrivé à pleine croissance', cf. russe rasti 'croitre', arménien ordi 'fils', hittite hardu 'petit-fils', latin arbor 'arbre', lat. arduus 'élevé, haut, escarpé ; difficile', irlandais ard 'haut' (d'où l'oronyme Ardennes, d'origine celtique). Rapproché de latin oriri 'surgir, se lever', ordo 'rang' (> fr. origine, ourdir, ordre) ; all. Art 'genre, espèce, façon', v.-iranien Arta 'le Juste-Ordre' (concept religieux de l'Avesta). Vrode donne lieu à des variantes populaires et/ou dialectales : navrode, navrod', vrodet'.

${ }^{4}$ Il existe en russe d'autres marqueurs (prépositions, locutions prépositionnelles) de sens proche, dont certains ont une origine liée à des schémas taxonomiques :

rod 'genre' $\rightarrow$ srodni + Dat. 'du genre de, proche de, ressemblant à';

$v i d$ 'espèce ${ }^{4} \rightarrow v$ vide + Gén. 'sous (la) / en forme de' ;

kačestvo 'qualité' $\rightarrow$ vačestve + Gén. 'en qualité de, en tant que'

maner 'manière, façon' (empr. au français) $\rightarrow$ na maner + Gén. 'à la façon de qqn /qqch.' ;

podobie 'façon, ressemblance' $\rightarrow$ napodobie + Gén. 'à l'instar de'.

${ }^{5}$ Mais même dans un contexte où Petuški serait le nom d'un grand bourg («qui n'est pas une ville mais qui ressemble à une ville »), l'énoncé (b) serait contraint. Pour le débloquer, il faudrait introduire le pronom indéfini čto-to : Selo Petuški-čto-to vrode goroda 'Le village de Petuški est une sorte de ville / est presque une ville'. En revanche, cf. la normalité de Petuški - gorod 'Petuški est une ville' ('Petuški est incluse dans la classe des villes'), énoncé certes banal. Notons aussi que Petuški - tipa goroda serait plus acceptable, car cet énoncé fait penser notamment à l'expression administrative posëlok gorodskogo tipa 'agglomération de type urbain'.
} 
proche d'une particule exprimant l'incertitude ou une valeur modale similaire.

\subsection{VRODE PRÉPOSITION}

Vrode (+ cas génitif) est une préposition qui présente deux types de constructions: a) GN1 vrode GN2 (Gén.) ; b) GV vrode GN (Gén.), avec possibilité de détachement syntaxique : GN1, vrode GN2, ... / $\mathrm{GV}$, vrode $\mathrm{GN}$.

En diachronie $\left(\mathrm{XVIII}^{\mathrm{e}}-\mathrm{XIX}{ }^{\mathrm{e}}\right.$ siècles), les contextes fréquents pour les marqueurs $v$ rode / vrode, dont les premières attestations datent de la $1^{\text {re }}$ moitié du XVIII ${ }^{\mathrm{e}}$ siècle, étaient liés à la notion de «genre » (au sens littéraire, artistique) : « création (littéraire, etc.) », « créer qqch. dans le genre / le style de ». V rode / vrode (la graphie attachée ne s'impose que progressivement) était sans doute calqué sur la construction française dans le genre de. Cf. :

(4) Éta kartina možet služit' obrazcom v rode izjaščnyx izdelij (revue Atenej, 1828) ${ }^{6}$

- Ce tableau peut servir de modèle dans le genre / dans la catégorie des belles créations.

$V$ rode / vrode pouvait dépendre d'un verbe :

(5) Vo vremja ego sekretarstva ona [Ekaterina II] ne raz prašivala ego pisat' vrode Felicy. (V. Xodasevič, Deržavin)

- À l'époque de son service en tant que secrétaire [auprès de la tsarine Catherine II], elle [Catherine II] lui demandait souvent d'écrire dans le genre de «Felitsa ».

(6) Vmesto al'manaxa ne zatejat' li nam žurnala v rode Edimburgh Review?

- Plutôt qu'un almanach, ne devrions-nous pas lancer une revue dans le genre de la Edimburgh Review ? (A. Puškin, 1830)

Dès le début, on observe une évolution vers des emplois semi-grammaticalisés et grammaticalisés (dont certains décrits par Čerkasova 1967) :

(7) Ja sdelal pis'mennyj akt vrode zaveščanija, po kotoromu daril v pol'zu vladimirskij gimnazii navgda vse moi sočinenija (I. Dolgorukov, 1788)

- J'ai rédigé un acte écrit, un genre de / une sorte de testament, dans lequel je léguais tous mes écrits au lycée de Vladimir.

(8) On lečilsja ot kakoj-to udivitel'noj bolezni, vrode katalepsii (A. Puškin, 1835)

- Il se faisait soigner d'une étrange maladie, du genre de / une sorte de catalepsie.

GN1 pouvait être syntaxiquement dissocié de GN2, et $v$ rode / vrode pouvait dépendre à la fois du GN1 (dans l'exemple suivant, du substantif karaul 'escorte') et du GV (dans l'exemple suivant, du participe présent actif du verbe naxodit'sja 'se trouver') :

(9) Gotov byl karaul iz reguljarnyx vojsk, naxodjaščixsja pri šaxe, v rode gvardii (A. Ermolov, 1817)

- Une escorte était constituée à partir des troupes se trouvant auprès du shah, en qualité de / en guise de garde / comme une sorte de garde.

Certains emplois se situaient hors toute relation de type taxonomique. Vrode y fonctionne clairement comme une préposition de comparaison métaphorique :

(10) ščëki, vrode antonovskix jablok (I. Turgenev, 1848)

- des joues comme / ressemblant à des pommes Antonovka.

(11) Ja pristal k nemu vrode teni (A. Griboedov, 1825)

- Je l'ai suivi comme une ombre / à la manière d'une ombre / comme si j'étais son ombre [vrode dépend ici d'un prédicat verbal].

Les emplois modernes (seconde moitié du $\mathrm{XX}^{\mathrm{e}}$ siècle, début du $\mathrm{XXI}^{\mathrm{e}}$ siècle) de vrode préposition se laissent répartir en quatre groupes : 1) approximation, 2) comparaison modalisée, 3) exemplification intensionnelle, 4) exemplification extensionnelle.

\subsubsection{Approximation : 'une sorte de, une espèce de'}

Dans ces contextes, on range N1 sous le même hyperonyme implicite que N2; N1 tend à être plus générique que N2. Deux constructions sont possibles : la construction non prédicative (la plus fréquente) et la construction prédicative (relativement rare), cf. :
(12) derevo vrode arbre
duba 'un arbre qui ressemble à un chêne, une sorte de chêne'
(13) Èto derevo
dans le genre
chêne (Gén.)
/construction non prédicative/
Cet arbre
vrode
duba
(copule zéro) dans le genre
chêne (Gén.)'

\footnotetext{
${ }^{6}$ Pour ne pas alourdir la présentation, nous indiquons les références des exemples de façon simplifiée. Si aucune référence n'est indiquée, cela suppose que l'exemple provient d'un des dictionnaires contemporains de la langue russe .
} 
'Cet arbre ressemble à un chêne / est du genre chêne / est proche du chêne /construction prédicative/

Voici des exemples qui illustrent la construction non prédicative :

(14) On napisal čto-to vrode romana, obeščal pokazat'

- 'Il a écrit qqch. du genre roman / une sorte de roman, il m'a promis de me le montrer';

(15) Pozdnij son vsegda predstavljalsja ej kakim-to besčinstvom, vrode vorovstva. Nu čto že, ugovarivala ona sebja, zaslužennyj otdyx. (I. Grekova)

- Elle avait toujours considéré la grasse matinée comme une action répréhensible, une sorte de vol. Elle essayait de se faire une raison : mais enfin, je l'ai bien mérité.

1.1.2. Comparaison modalisée : '(un peu) comme, à la façon de'

(16) Xarakter u neë byl vrode tvoego, takoj že vspyl'čivyj

- 'Son caractère était du genre du tien / comme le tien / proche du tien, tout aussi impulsif' /construction prédicative/;

(17) My poterjali ne tol'ko sovetskoe graždanstvo, no i svoju ètničeskuju, istoričeskuju, povedenčeskuju prinadležnost'. My, predstaviteli strašnogo i mogučego SSSR, stali kroxotnym nacional'nym men'šinstvom. Vrode narodov Krajnego Severa. (P. Vajl, A. Genis)

- Nous avons perdu non seulement notre citoyenneté soviétique, nous avons perdu notre identité ethnique, historique, comportementale. Nous, représentants de la redoutable et puissante URSS, sommes devenus une minuscule minorité ethnique. Un peu comme les peuples du Grand Nord.

Exemple qui illustre la construction prédicative :

(18) Anfisa Gromova byla mne vrode sestry - bogodannoj, sud'bodannoj, - xotja i ssorilis' my žestoko i podolgu byli počti vragami (I. Grekova) /construction prédicative/

- Anfisa Gromova était pour moi un peu comme une sœur / une sorte de sœur, qui m'avait été donnée par Dieu, par le destin, - même s'il nous arrivait de nous disputer violemment et d'être presque des ennemies pendant de longues périodes.

Vrode peut dépendre immédiatement d'un adjectif :

(19) Obama - kloun ; a ja dumala, čto on sportivnyj $i$ šustryj, vrode Putina... (forum sur Internet)

- Obama est un clown; et moi, je croyais qu'il était sportif et dynamique, un peu comme Poutine.

1.1.3. Exemplification intensionnelle : 'tels que'

(20) Ostrosjužetnyj, to est' dinamičnyj, gde uže na pervoj stranice svoračivajut skulu: èto vam ne logičeskie krossvordy dobryx staryx angličanok vrode Agaty Kristi. (S. Džimbinov)

- Un sujet captivant, autrement dit sujet dynamique, où le personnage se fait casser la figure dès les premières pages : on est loin des énigmes logiques des bonnes vieilles Anglaises du genre d'Agatha Christie.

(21) Mnogie ženščiny mečtajut o mužčinax vrode Putina. A kto že togda poljubit Pitinyx i Bezputinyx ? (forum sur Internet, 2014)

- De nombreuses femmes rêvent d'hommes du genre de / tels que Poutine. Et quelles sont celles qui vont tomber amoureuses des Pitines et des Bezpoutines ${ }^{7}$ ?

1.1.4. Exemplification extensionnelle : 'notamment', 'comme par exemple', 'à savoir'

(22) Vse byli soglasny s brigadirom. Bolee togo, vse byli vozmuščeny, a inye, vrode Kol'ki Zabalueva, daže oskorbljalis' i grustno, gor'ko vzdyxali. (V. Šukšin)

- Tout le monde était d'accord avec le chef d'équipe. De plus, tous étaient indignés, et certains, comme par exemple Kolka Zabaluev, se montraient même vexés et poussaient des soupirs pleins de tristesse et d'amertume.

(23) Vy verite, čto u vidnyx politikov vrode: Putina, Obamy, Fidelja Kastro, Angely Merkel' - est' dvojniki ? (forum sur Internet, 2014)

- Vous croyez vraiment que les chefs politiques tels que / comme par exemple : Poutine, Obama, Fidel Castro, Angela Merkel, - ont des sosies?

Les contextes du groupe (1.1.3) ne sont pas toujours faciles à distinguer des contextes du groupe

\footnotetext{
${ }^{7}$ Jeu de mots : en russe, Pitin se comprend comme « celui qui boit », Bezputin / Besputin se comprend comme « celui qui mène une vie dépravée ».
} 
Dans les emplois de ce type, vrode signifie 'apparemment', 'comme si', 'on dirait', 'soi-disant'. Parmi les synonymes relatifs en russe: vrode by, budto, kak budto, kak budto by, kažetsja, poxože. Combinaisons possibles avec d'autres mots modaux: vrode by, vrode kak, vrode by kak, vrode kak by. Pour plus de détails, voir les exemples et les analyses dans nos travaux (Sakhno 2002, Sakhno 2010).

1.2.1. Prise en compte par l'énonciateur (So) d'une incompatibilité ( $P$ et non- $P$ à la fois) : 'apparemment', 'on va dire'

(24) - Vot vrode vrozlyj čelovek, sem'ja u tebja, na zavode tebja uvažajut, a prostyx veščej ne ponimaeš'. Kakix? - Net, ne ponimaeš'. U tebja že syn! (I. Alekseev 134)

- Tu es un homme apparemment / on va dire adulte, tu as une famille, on te respecte dans ton usine, mais tu ne comprends pas les choses les plus simples. - Quelles choses ? - Non, tu ne comprends pas. Tu es père d'un fils!

L'une des traductions de vrode que nous proposons ici en français (on va dire) tient compte du fait que So ne met pas vraiment en doute le statut de l'interlocuteur en tant qu'adulte, mais il signale l'incompatibilité entre la propriété «être adulte » et le comportement «irresponsable » de l'interlocuteur.

1.2.2. Illusion, simulation : 'comme si', 'soi-disant'

(25) Na drugoj den' našego geroja vypustili iz izoljatora, pognali na lesozavod, a izoljator ne byl daže oformlen prikazom, vrode sidel, a vrode i net, nikakix sledov, posledstvij ne ostalos' v bumažnom xozjajstve lagerja. (E. Fëdorov)

- Le lendemain, on laissa notre héros sortir du mitard, et on l'expédia à la scierie, alors que l'incarcération au mitard ne fit même pas l'objet d'un arrêté : c'était comme s'il avait séjourné au mitard et comme s'il n'y avait jamais séjourné ; les documents officiels du camp n'en gardèrent aucune trace.

(26) Vit'ka prjamo tut že, za stolom celoval Ritu, a Rita slabo bila rukoj Vit'ku po pleču, vrode ottalkivala, a sama l'nula tugoj grud'ju i drugoj rukoj obnimala za šeju. (V. Šukšin)

- Vitka embrassait Rita sans quitter la table, Rita donnait des faibles coups de poing sur l'épaule de Vitka, comme si elle le repoussait, mais en réalité, elle serrait son opulente poitrine contre la sienne et enlaçait son cou de l'autre bras.

Dans ce dernier exemple, vrode fonctionne clairement comme connecteur.

1.2.3. Citation "non verbatim" à caractère polémique : 'il parât', 'soi-disant'

(27) Mif o zagranice načalsja s varjagov. Vrode u nix byl porjadok. Potom porjadok pojavilsja i doma. Poètomu zagranicu zapretili. Priznali eё nesuščestvujuscej, potom nenužnoj, zatem nepravil’noj. (P. Vajl, A. Genis)

- Le mythe de l'étranger remonte aux Varègues. Chez eux, il y avait, paraitt-il / soi-disant, de l'ordre. Ensuite, on a introduit de l'ordre chez nous. C'est pourquoi l'étranger est devenu un tabou. On l'a déclaré d'abord inexistant, plus tard inutile, puis politiquement incorrect.

1.2.4. Inférence, interprétation et référence implicite à la parole de l'autre : 'il paraît que'

(28) - Rybëška est'v reke? - Malo. Rebjata vverx zaplyvajut, tam vrode polučše. (V. Šukšin)

- Y a-t-il des poissons dans la rivière ? - Peu. Les gars s'en vont pêcher en amont, ça mord un peu mieux à cet endroit, il paraît.

1.2.5. Non-prise de position (réponse évasive)

(29) - Ty zdorov? - Vrode /Vrode zdorov /Zdorov vrode /Vrode da / Da vrode

- '- Ta santé, ça va ? - Oui, ça a l'air d'aller / on va dire que ça va'.

(30) Èto pravda, čto vaš institut likvidirujut? - Vrode.

- 'Est-il vrai que votre institut sera fermé ? - Il parât'

Dans ce dernier contexte, il y a aussi une référence implicite à la parole de l'autre ou des autres, ce qui le rapproche des cas du type 1.2.3 et 1.2.4.

\section{CONTEXTES TRANSITOIRES : ENTRE VRODE PRÉPOSITION ET VRODE MOT MODAL}

Dans certains contextes modernes, la limite entre vrode préposition et vrode mot modal n'est pas très nette. Cf. :

(31) Javilsja raskonvojnik - djadja s odnoj derevjannoj nogoj, prisobačennoj $k$ kul'te verëvkami. 
Vybravšis' na volju, on skakal daže kak-to ozorno - ne kak invalid, a vrode mal'čonkoj (Instr.). (A. Pavlov) - Le responsable d'escorte est arrivé : c'était un bonhomme qui avait une jambe de bois, fixée à son moignon avec des cordelettes. Retrouvant le grand espace, il trottinait (m. à m. sautillait) avec une sorte d'agilité, non pas comme un invalide, mais pour ainsi dire comme un gamin.

La construction On skakal (...) vrode mal'čonkoj 'Il sautillait à la façon d'un gamin / comme s'était gamin' où vrode ne régit pas grammaticalement mal'čonkoj qui est le cas instrumental de mal'čonka 'gamin' et qui fonctionne comme un adverbe de manière ('à la façon d'un gamin'), impose la lecture de vrode comme mot modal ('pour ainsi dire'). Mais si on tient compte du style de ce texte qui reflète les tendances du russe familier, on peut considérer cette séquence comme un «mixte » de deux constructions :

.... skakal vrode mal'čonkoj (Instr.) '(il) sautillait à la façon de / pour ainsi dire comme un gamin ' .... skakal vrode mal'čonki (Gén.) '(il) sautillait à la façon / dans le genre d'un gamin'.

Dans la seconde construction, vrode est une préposition régissant le cas génitif.

Voici un exemple du même ordre, mais ici vrode fonctionnant en tant que mot modal introduit un GN au cas nominatif :

(32) Kto-to skazal ej, čto u Ljusi deduška rabotaet dekanom v tom samom institute, kuda postupal Vadim. A dekan - èto vrode bol'šoj načal'nik (Nom.). I rešila Anfisa tajkom ot Vadima k tomu dekanu s"ezdit' (I. Grekova)

- Quelqu'un lui avait dit que le grand-père de Lussia était le doyen de la faculté où Vadim voulait s'inscrire. Et un doyen, c'est pour ainsi dire /, semble-t-il, un grand chef. Anfisa décida donc d'aller voir ce doyen, sans rien dire à Vadim.

On pourrait très bien paraphraser la séquence en mettant le GN introduit par vrode au cas génitif : A dekan - èto vrode bol'šogo načal'nika (Gén.)... 'Et un doyen, c'est une sorte de grand chef' (on notera que N1 est plus spécifique que N2, contrairement aux exemples 12-14).

Et il est possible de considérer ce type de contextes comme le résultat d'une neutralisation de la marque casuelle du génitif et / ou comme une sorte de glissement du rapport de prédication vers un rapport de dénomination.

\section{TIPA : DE LA TAXONOMIE «ABSTRAITE » À LA MODÉLISATION GLOBALISANTE DU DIRE}

Le marqueur russe tipa «du type de » peut fonctionner d'une part comme une préposition à sens véritablement taxonomique (tkan' tipa sitca 'un tissu du type indienne') et d'autre part, comme une préposition de «comparaison modalisée» (politiki tipa Putina 'des hommes politiques tels que / semblables à Poutine'), mais aussi comme une particule modale discursive de caractère métalinguistique, avec des effets de sens proches de 'pour ainsi dire, en quelque sorte; soi-disant'.

\subsection{TIPA PRÉPOSITION (COMPARAISON MODALISÉE)}

Les exemples sont très nombreux dans le discours russe d'aujourd'hui. Dans plusieurs cas (comme dans l'exemple 33), le remplacement par vrode est possible. Cf. :

(33) U Kabineta dostatočno ryčagov dlja blokirovanija v parlamente oprometčivyx šagov tipa vydelenija neskol'kix desjatkov trillionov, ne predusmotrennyx bjudžetom (journal Izvestija)

- Le Cabinet des ministres dispose de moyens suffisants pour bloquer au parlement toute décision irréfléchie, du type de l'attribution de plusieurs dizaines de trillions de roubles non prévus par le budget.

(34) Odnako drugie strany, upravljaemye sil'nymi liderami tipa Putina, mogut posčitat' èto xorošej ideej (forum sur Internet, 2014)

- Pourtant d'autres pays, qui sont dirigés par des leaders forts du type de / tels que Poutine, peuvent considérer qu'il s'agit d'une bonne idée.

Le détachement syntaxique (marqué par une virgule à l'écrit) n'est pas rare, surtout dans des contextes proches de «exemplification extensionnelle »:

(35) Georgievskie lentočki nosjat tol'ko otpetye idioty, tipa Putina i Medvedeva (forum sur Internet, 2014) - Les rubans Saint Georges sont arborés seulement par des idiots accomplis, du type de Poutine et de Medvedev.

Les exemples 34 et 35 semblent très proches des exemples 21 et 22 vus supra (avec vrode), mais on peut noter que vrode, à la différence de tipa, implique plus directement une inclusion inconditionnelle de $X$ (exemple 21 : «Poutine») dans la classe $Y$ (exemple $21:$ : les hommes à image positive »), en rapport avec le prédicat principal (exemple $21:$ : rêver »), selon le principe «parler de $X$ signifie en quelque sorte parler de la classe $Y »$. Ce serait l'effet de sens que vrode induirait dans $(34,35)$ si on le substituait à tipa.

Dans le russe oral d'aujourd'hui, le GN introduit par tipa peut rester au cas nominatif (cf. les 
exemples 31, 32 analysés ci-dessus, avec vrode) :

(36) Vot ona i eščë kakoj-to parniška. Tipa prodjuser. Vot on ë̈ snačala xotel raskrutit' kak pevicu. (Ruscorpora, corpus oral, 2005)

- La voici avec un gars. Type / Genre producteur en show-biz. Lui, il voulait au début la lancer en tant que chanteuse.

\subsection{TIPA MARQUEUR DISCURSIF (RUSSE ORAL FAMILIER)}

À la différence de vrode, tipa ne marque pas l'incertitude de l'énonciateur concernant la prédication qu'il modalise. La modalisation ne porte pas sur le fait exprimé par l'énoncé, mais sur l'expression ellemême, sur la dénomination d'un événement ou d'un élément de la réalité, ou sur la façon dont l'énonciateur rend le discours de l'autre en le citant ou son propre discours antérieur au moment de l'énonciation.

2.2.1. TIPA marqueur de modalisation métalinguistique

(37) Ego tipa uvolili 'Il a été pour ainsi dire licencié' [le locuteur admet que l'expression licencier est inexacte].

Cet emploi récent est certes lié au style très familier jugé « relâché » par les locuteurs russophones cultivés. Un Russe cultivé utiliserait dans ce cas une autre particule : kak by 'en quelque sorte, comme si' (qui existe depuis longtemps, quoiqu'elle ait souvent été stigmatisée, car considérée comme un tic de langage) : Ego kak by uvolili. Avec vrode, l'énoncé aurait un sens différent :

(37') Ego vrode uvolili 'Apparemment, a été licencié' [le locuteur n'est pas sûr du fait « il a été licencié », ou il se distancie de la source de cette information].

2.2.2. TIPA marqueur de citation non-verbatim et marqueur d'une modélisation globalisante du dire ('genre', 'on va dire')

À la différence de vrode (voir 1.2.2), cet emploi de tipa n'est pas lié aux effets de sens tels que «illusion, simulation» ('comme si', 'soi-disant'). La meilleure traduction en français serait genre (dans son emploi oral particulier récent) :

(38) Nu, ona tipa skazala, čto ja s nej ne xoču bol'še vstrečat'sja iz čuvstva mesti [Ruscorpora, corpus oral 2005]

- Eh bien, elle a genre dit que je ne voulais plus la voir, par sentiment de vengeance.

(39) Zato vyjdu zamuž skorej vsego po ljubvi / potomu čto ona posmotrela na moi linii i skazala / čto tipa odna tam vot u menja linija kakaja-to tolstaja [Ruscorpora, corpus oral, 2006]

- En revanche, je ferai probablement un mariage d'amour, parce qu'elle [la chiromancienne] a regardé mes lignes [de la main] et elle a dit genre que j'ai une ligne qui est plutôt épaisse.

(40) Ix tipa na podušečku kladut / čtob lučše vidno bylo i čtob osveščalis’ ravnomernej [Ruscorpora, corpus oral, 2005]

- On genre les met sur un coussin, pour qu'on les voie mieux et pour qu'ils soient éclairés de façon plus harmonieuse [présentation prestigieuse qui permet de mettre en valeur les bijoux dans les magasins de joaillerie].

(41) - A ty kušaeš ? - Nu tipa togo. A ty ? [Ruscorpora, corpus oral, 2005]

- Alors, tu es en train de manger? - Oui, genre (m. à $\mathrm{m}$. du type de cela). Et toi ? [conversation téléphonique entre deux étudiants].

\section{ASPECTS TYPOLOGIQUES: MARQUEURS ANALOGUES À VRODE / TIPA DANS D'AUTRES LANGUES}

À titre de parallèle sémantique à russes vrode et tipa, on peut penser d'abord au mot français genre dans ses emplois oraux particuliers, apparus au début des années 1980 (emplois analysés par DanonBoileau, Morel 1997, Yaguello 1998, Fleischman 2006, 2007, Fleischman, Yaguello 2004), avec d'une part les sens 'quelque chose comme' ou 'plutôt' : un bonhomme genre homme d'affaire; Elle est genre méchante avec certains élèves, cette prof; et d'autre part, avec un sens proche de 'à peu près' : Y en a genre $50 \%$ qui ont voté contre. Genre peut même exprimer une confirmation évasive : - Il a fait une dépression, c'est bien ça? - Genre.

Par ailleurs, genre fonctionne souvent comme un connecteur discursif (Yaguello 1998 : 18-24), il permet notamment au locuteur de reformuler son énoncé, ou bien de rapporter les paroles attribuées à autrui - et non pas réellement prononcées - tout en s'en dissociant (sens proches de 'comme si', 'c'est-à-dire, je veux dire par là') : Elle me demande tout le temps de l'aider, genre elle n'a rien compris au cours ('comme 
si elle n'avait rien compris' ou 'c'est comme si elle disait : «je n'ai rien compris au cours »'), Ce jean, il me va mieux, genre il me serre déjà moins ${ }^{8}$.

Il en est de même de fr. type, style dans leurs emplois oraux modernes, cf. Les arômes que ressort euh ce vin on sent euh des odeurs de cassis des odeurs euh de fruits évolué(e) type griotte euh fruits confits ; Donc en fait le matin (...) est-ce que vous avez un café d'accueil ? Du style euh je ne sais pas croissant euh pain au chocolat; C'est un mec genre style zonard 'He's kinda like a dropout' (exemples empruntés à Fleischman 1999, Mihatsch 2006).

Cependant, russes vrode et tipa ne semblent pas évoluer vers des emplois analogues à celui de fr. genre, lorsque ce dernier introduit une interprétation que l'énonciateur fait d'une citation « verbatim » de la parole de l'autre : Il me dit: "Tu vas te planter au brevet," genre que je vais me laisser impressionner 'He says to me: "You're gonna screw up on the brevet exam." Like I'm going to be upset by that!' (exemple emprunté à Fleischman 1999, avec sa traduction en anglais familier). Dans ce contexte, vrode / tipa seraient contraints.

En anglais, le lexème kind 'genre' est un classificateur, conformément à son sens lexical premier (this kind of book 'ce genre de livre'), mais il fonctionne comme approximateur dans There was a kind of tinking sound ' On entendait quelque chose qui ressemblait à un bruit de grelot', et la structure kind of peut devenir un modalisateur discursif dans He was kind of worried-looking 'Il avait l'air comme qui dirait inquiet', I kind of like that 'J'aime assez ça', ou marquer une réponse évasive dans : - Aren't you pleased ? - Kind of! '- Tu n'es pas content ? - Si, assez!'.

En latin, quasi se comportait en tant que connecteur conditionnel ('comme si, comme') et en tant que approximateur ('environ', 'une sorte de', 'presque', à peu près', 'pour ainsi dire'). Certaines de ces valeurs se perpétuent dans italien quasi qui développe par ailleurs des emplois discursifs plus complexes (quasi, redoublé quasi quasi : modalisateur d'énoncé), cf. Et allora, io quasi quasi prendo il treno et vengo, vengo da te 'Et alors, franchement, je pense prendre le train et venir, venir chez toi' (A. Celentano, Azzurro, 1960, cit. d'après Bertocchi, Maraldi, Orlandini 2014) ${ }^{9}$. Portugais tipo et italien tipo fonctionnent (usage familier) comme approximateurs, cf. ital. Egli è venuto tipo dieci minuti fa 'Il est venu environ / fam. genre il y a 10 minutes ${ }^{10}$.

D'autres parallèles sémantiques peuvent être observés dans différentes langues du monde. Ainsi, plusieurs langues réalisent le passage du sens lexical ou semi-lexical «simile » ('similitude, ressemblance') vers la catégorie fonctionnelle grammaticale «quotative», liée à la citation non littérale (angl. «nonverbatim quotative ») de la parole de l'autre ou de celle de l'énonciateur, citation qui peut être parfois plus ou moins fictive, simulée, cf. ces exemples cités d'après (Heine, Kuteva $2002: 274$ ) :

allemand oral, lexème so 'ainsi, de cette façon' (sens propre) : Ich sagte ihm, dass er gehen muss. Und er so, ich werde es mir überlegen 'Je lui ai dit qu'il devait y aller. Et lui, il me laisse entendre [comme quoi] que je dois y réfléchir' ;

anglais oral (USA), lexème like 'comme' (sens propre) : And I'm like : Gimme a break, will you! 'Et moi, je lui dis un truc genre [comme quoi] «Laisse-moi tranquille! »' (voir également les travaux de S. Fleischman 1998, 1999);

finnois, lexème niinku 'comme' (sens propre) : Ja sit mä olin niinku että herrajjumala et voi olla totta 'Et alors, j'étais dans un état genre [comme quoi] «Mon Dieu, je ne peux pas y croire !»' ;

kxoe (groupe de langues khoin, Afrique du Sud) : taá 'être comme (ça)', 'ainsi' (sens propre) > marquer de discours indirect: Mà-ka tca kuùn-wà-goè taá ti'oa-ra-han 'Je t'ai demandé [comme quoi] où tu allais'.

\section{CONCLUSION}

La polyfonctionnalité du morphème russe vrode se présente comme une dynamique inscrite dans un processus de grammaticalisation, avec un cheminement sémantique complexe, considéré à la fois en diachronie et en synchronie, qu'on peut schématiser comme ceci :

$V R O D E:$ «procréation, naișsance » [sens diachronique, étymologie : racine -rod-]

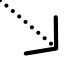

\footnotetext{
${ }^{8}$ M. Yaguello note que chacun des emplois modernes de genre renvoie implicitement à une norme, soit sous la forme d'un calibrage, soit sous la forme d'un recadrage du dire, avec toujours à l'arrière-plan la présence de l'énonciateur qui fait entendre sa voix pour formuler un jugement.

${ }^{9}$ Voir la contribution de A. Bertocchi, M. Maraldi, A. Orlandini dans le présent volume.

${ }^{10} \mathrm{Je}$ remercie mes collègues romanistes de l'U. Paris Ouest pour ces données sur le portugais et l'italien, en particulier M. Giuseppe Gargiulo, MCF HDR, pour la traduction de l'exemple italien avec quasi quasi (les éléments qui rendent l'effet de sens de ce marqueur, difficile à traduire en français, sont en italique dans la traduction).
} 
« généricité » [sens synchronique fédérateur, cf. lexème rod]

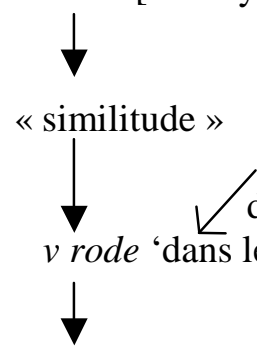

+ «localisation » [apport sémantique

de la préposition $v+$ cas locatif]

$\checkmark$ rode 'dans le genre' (d'abord locution libre,

$\downarrow \quad$ ensuite semi-figée et figée)

(niveau du GN : vrode préposition)

«approximation» « comparaison » « exemplification»

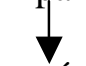

(niveau de l'énoncé : vrode particule, quasi-adverbe modal)

modalité épistémique ('apparemment' et autres sens modaux de ce type)

(niveau de l'énonciation: vrode particule, mot modal discursif)

divers sens modaux liés aux rapports intersubjectifs (« citation»; «simulacre», etc.)

Une étape ultérieure est d'ailleurs envisageable, celle de la désémantisation, où vrode deviendrait un « mot parasite » : ainsi (Bulygina, Šmelëv 1997 : 304) signalent que vrode particule tend à être en partie désémantisé dans le russe oral, tout en restant un «quotatif » qui marque la grammaticalisation des valeurs liées à l'énonciation «médiatisée » (ce qui correspond au terme anglais evidentiality).

Les sens ainsi définis ne sont pas toujours parfaitement délimités, mais constituent un continuum : ainsi, la limite entre «approximation» et «comparaison», et d'autre part, entre «comparaison» et «exemplification»n'est pas absolument nette. Mais «comparaison» semble une étape sémantique qui génère d'une part «approximation » et d'autre part, « exemplification ».

Quant au morphème russe tipa, son sens de base en synchronie se laisse définir en termes de «modèle », « exemple », idée qui découle de «marque imprimée par un coup, empreinte » (étymologie du mot grec source typos, avec un développement sémantique en grec vers «figure, image ${ }^{11}{ }^{1}$ ). Le sens « modèle » donne lieu aux sens « comparaison» et «exemplification» qui sont présents en russe au même titre à cette étape de la grammaticalisation. Ce sens fédérateur («modèle ») se retrouve dans le principe sémantique du fonctionnement de tipa en tant que particule intervenant au niveau de l'énonciation, car tipa marque une «modélisation globalisante » du dire : soit dans la description par l'énonciateur d'une situation, soit dans la manière dont l'énonciateur rapporte le dire d'autrui ou son propre dire (effectué antérieurement).

Voici une représentation schématique du parcours sémantique de tipa, qui aboutit à sa grammaticalisation allant jusqu'à la désémantisation (tipa en tant que tic de langage, mot parasite, speech filler) :

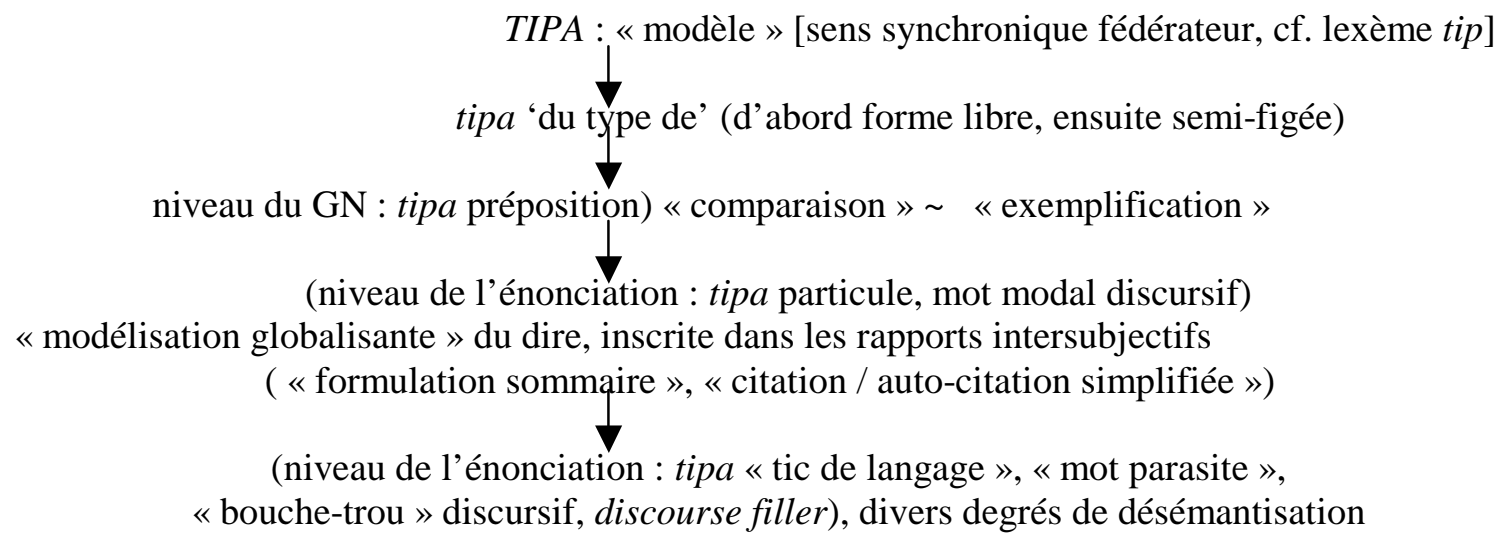

En particulier, vrode et tipa illustrent, chacun à sa façon, le passage du sens lexical ou semi-lexical « simile «(similitude, ressemblance) vers la catégorie fonctionnelle grammaticale « quotative » (citation),

\footnotetext{
${ }^{11} \mathrm{Ce}$ dernier sens n'est pas pertinent en synchronie pour le lexème russe tip, à la différence de vrode, où rod lié à « naissance » est toujours ressenti par les locuteurs.
} 
plus exactement à «nonverbatim quotative » (citation non littérale de la parole de l'autre, ou de la parole de l'énonciateur lui-même, qui peut être parfois plus ou moins fictive, simulée) ${ }^{12}$.

\section{Bibliographie}

Archaimbault S., Les premières grammaires du slavon, in: S. Auroux (dir.), Histoire des idées linguistiques, t. 2 : Le développement de la grammaire occidentale, Liège, Madraga, 1992, p. 239-250.

Archaimbault S., Préhistoire de l'aspect verbal: L'émergence de la notion dans les grammaires russes. Paris, CNRS Editions, 1999.

Bertin A., Bat-Zeev Shyldkrot H., Soutet O. (dir.), Langages 190, 2013, « Subordonnants et subordination : frontières, chevauchements et cas limites $»$.

Bertocchi A., Maraldi M., Orlandini A. Quasi : du latin à l'italien, - Communication au colloque international Prépositions, conjonctions et connecteurs, Paris, 14-15.03 2014.

Bulygina, T. V., Šmelëv A. D., Jazykovaja konceptualizacija mira (na materiale russkoj grammatiki), Moskva, Jazyki russkoj kul'tury, 1997.

Čerkasova E. T., Perexod polnoznačnyx slov v predlogi, Moskva, Nauka, 1967.

Danon-Boileau L., Morel M.-A., Question, point de vue, genre, style...: les noms prépositionnels en français contemporain, Faits de langues, 9, 1997, p. 193-200.

Fleischman S., Des jumeaux du discours : genre et like, La linguistique, 34, 2, 1998, p. 31-47.

Fleischman S., Pragmatic markers in comparative perspective: A contribution to cross-language pragmatics Paper presented at Pragma 99, Tel Aviv, June 1999.

Fleischman S., Yaguello M., Discourse markers across languages ? Evidence from English et French, - in C. Moder, A. Martinovic-Zic (eds), Discourse analysis across languages and cultures, Amsterdam, Benjamins, 2004, p. 129-147.

Heine B., Kuteva T., World lexicon of grammaticalization, Cambridge, New York, Cambridge Univ. Press, 2002.

Lakoff G., Hedges : A study in meaning criteria and the logic of fuzzy concepts, Journal of Philosophical Logic, 2, 4, 1973, p. 458-508.

Mihatsch W., Espèce de, sorte de, genre de: des marqueurs d'approximations entre sémantique et pragmatique, Revue de sémantique et de pragmatique, 19-20, 2006, 229-248.

Mihatsch W., The construction of vagueness : 'Sort of' expressions in Romance languages, in G. Radden, K.-M.Koepcke et al. (eds), Aspects of meaning constructing meaning : from concepts to utterances, Amsterdam, Benjamins, 2007, p. 225-245.

Mihatsch W., The approximators French comme, Italian come, Portuguese como, Spanish como from a grammaticalization perspective, in C. Rossari et al. (eds), Grammaticalization and pragmatics, Bingley, Emerald, 2009, p. 65-92.

Mihatsch W., Les approximateurs quantitatifs entre scalarité et non-scalarité, Langue Française, 165, 2010, p. $125-154$.

Ruscorpora 2003-2014 - Национальный корпус русского языка (Nacional'nyj korpus russkogo jazyka), www.ruscorpora.ru.

Sakhno S., Dictionnaire russe-français d'étymologie comparée. Correspondances lexicales historiques. Paris, L'Harmattan, 2001, p. 331-354.

Sakhno S., Opyt opisanija slova vrode : uslovnaja prototipičnost'. - Rusistika segodnja, 2002, 1-4, p. 45-57. Sakhno S., Les avatars du sens et de la fonction dans le phénomène de la grammaticalisation. Description systématique du lexème russe vrode "dans le genre de » comparé à d'autres lexèmes russes grammaticalisés à fonctionnement proche, http : //hal.archives-ouvertes.fr/hal-0076537, 2012, 210 pages.

Yaguello M., Petits faits de langue, Paris, Seuil, 1998.

SAKHNO Serguei,

U. Paris Ouest Nanterre, MoDyCo

\footnotetext{
12 On constate une coalescence sémantique remarquable entre l'un des effets de sens du grec ancien typos (typô legein 'dire sommairement') et la glose que nous avons pu établir pour l'un des emplois de tipa particule («modélisation globalisante du dire »).
} 